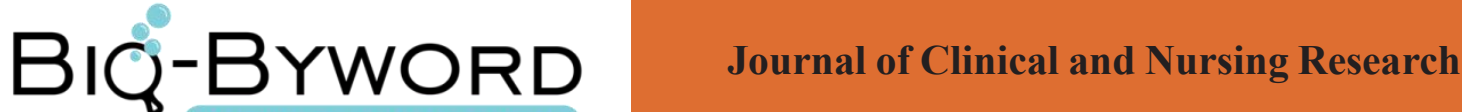

\section{Analysis on Safety Management of Nursing Drugs in Cardiology}

\author{
Chen Tian Tian
}

New Hospital of Xinyi City, Jiangsu Province, Xinyi 221400

\section{ARTICLE INFO}

Article history:

Published online: $30^{\text {th }}$ Sept, 2017

\section{Key words:}

Cardiology

Nursing medication

Safety management measures

Treatment effect

\begin{abstract}
Objective: To manage the safety management of nursing drugs. Methods: 120 patients were randomly divided into two groups: the management group and the control group. The control group was treated with routine medication. The management group was treated with drug safety management on the basis of the management group. And the values of the two groups were compared. Results: The treatment effect and nursing satisfaction were higher in the management group than in the control group, and the error rate was lower than that of the control group $(\mathrm{P}<$ 0.05). Conclusion: Nursing medication in patients with cardiology in nursing care to take safety management measures. It can strengthen the treatment of patients and nursing effects, worthy of promotion.
\end{abstract}

\section{Introduction}

The current clinical, most of the treatment for patients with cardiology is the use of drugs for treatment, so the patient's medication is correct and safe, it becomes an important factor in the recovery of patients in the course of care medication, at any time may be Because the workload of nursing staff is too large, or the operation process is not standardized, leading to drug safety problems, triggering the patient's physical crisis, and even medical disputes ${ }^{[1]}$. Therefore, this article mainly for the heart of the nursing work in the implementation of nursing drug safety management measures to analyze the effect.

\section{Information and methods}

\subsection{General information}

The patients were randomly divided into two groups: the management group and the control group, each group of 60 patients, the management group of patients, the patients were randomly divided into two groups, the management group and the control group, each group of 60 patients, the management group of patients Aged between 37 and 75 years old, female patients in 25 cases, 35 cases of male patients, weighing $46.5-74.1 \mathrm{~kg}$, the average $(56.2 \pm 2.1) \mathrm{kg}$. , The control group of patients aged 39 to 79 years old, female patients in 21 cases, 39 cases of male patients, weight $45.6-80.3 \mathrm{~kg}$, the average $(45.4 \pm 4.6) \mathrm{kg}$. There were no significant differences in age, body weight, and other general data between the two groups of patients with cardiology $(\mathrm{P}>0.05)$. Both groups signed a study consent form.

\subsection{Research methods}

In the control group, 60 patients with cardiology were treated with routine nursing medication: 1) Follow the three pairs of seven pairs of patients to check the use of drugs. 2) Patients with oral medication in the patient to take care of, the infusion should regularly check the infusion situation, the problem in time with the physician to contact.

On the management group of 60 patients with cardiology patients on the basis of conventional care medication, the use of care drug safety management: 1) Drug classification and check: the hospital in the drug management, we must develop a strict drug classification and drug use check. The operation process and system of drugs must be placed neatly and detailed description of the situation of drugs. To check multiple time to avoid errors. 2) Nursing staff safety awareness: In the training of nurses, we must explain the importance of the safety of 
the use of drugs and the safe use of drugs for training. 3) The establishment of a sound system for the safe use of drugs: The establishment of a formal drug management mechanism, as well as the standardized use of drugs requiring nurses to strictly abide by the requirements for use. The development of scientific drug use inspection plan, as far as possible on information. The use of drugs, the patient's use of the situation to be checked, and to strengthen the management of injection drugs for the management and maintenance ${ }^{[2]}$

\subsection{Evaluation indicators}

The curative effect of the two groups of patients with cardiology and drug errors were observed statistically by the use of questionnaires to investigate the satisfaction of patients with care medication.

\subsection{Data processing}

The data of this study were analyzed by SPSS 17.0 software. The treatment effect, the rate of drug error and the satisfaction rate of medication were used as the counting data, expressed in the rate (\%) and the chi-square test. $\mathrm{P}<0.05$ that the difference was statistically significant.

\section{Results}

Management group of patients with treatment and nursing satisfaction were higher than the control group of patients, and medication error rate was significantly lower than the control group of patients. The difference was statistically significant $(\mathrm{P}<0.05)$. See Table 1 for details.

Table 1 Treatment effect, medication error events and nursing satisfaction [n (\%)]

\begin{tabular}{cccc}
\hline & Treatment efficiency & Error event & Nursing satisfaction \\
\hline Management group $(\mathrm{n}=60)$ & $56(93.00)$ & $1(2.00)$ & $59(98.00)$ \\
Control group $(\mathrm{n}=60)$ & $48(80.00)$ & $13(22.00)$ & $52(87.00)$ \\
$\mathrm{x} 2$ & 4.615 & 10.644 & Time \\
P value & $<0.05$ & $<0.05$ & $<0.05$ \\
\hline
\end{tabular}

Note: Compared with the control group, ${ }^{*} \mathrm{P}<0.05$

\section{Discussion}

Most patients with cardiomyopathy are more serious and the causes of the patients are also different. The treatment is also different because of the condition and the physical condition of the patients. However, most of them are still in the main method of drug therapy. In diversity, the use of the drug specifications, origin and so on are different and according to the patient's physical condition and the disease. The physician out of the drug dose and methods are different, so in use, it is easy to confuse the situation and use drug treatment. Once the use of drugs problems, not only will cause harm to the patient's body, but also lead to patients do not trust the hospital, there are medical disputes $^{[3]}$.

In recent years, the clinical safety of care for the more and more attention. In the conventional care drug mode, the implementation of nursing drug safety management measures requiring nurses to gradually improve their level of medication as well as the sense of responsibility to strengthen the drug of the relevant knowledge to learn and regulate the management system of drugs, as well as the operation of the drug process to strengthen the use of drugs when the inspection can effectively strengthen the safety of care medication. Improve the handling capacity of accidents. Once the patients use drugs, there is a problem, in time with the responsible physician contact ${ }^{[4]}$.

\section{Conclusion}

In summary, in the care of patients with cardiology, the use of care medication safety management measures can effectively improve the patient's treatment and nursing satisfaction, reduce the probability of occurrence of medication errors, in order to strengthen the treatment of patients and care effect.

\section{References}

[1] Zhang Xiumei. Cardiology Nursing Drug Safety Management Analysis. Chinese Journal of Traditional Chinese Medicine, 2016, 10(3): 68-70.

[2] Wang Yan. Cardiology Nursing Drug Safety Management Clinical Exploration Analysis. Northern Medicine, 2013, 2(9): 74.

[3] Wu Fengjia, Jin Jianfen, Sun Hongjuan. Clinical Analysis of the Safety Management of Cardiology Medication. Journal of Traditional Chinese Medicine and Pharmacology, 2017, 2(13): 82-83. 